Foss. Rec., 21, 159-169, 2018

https://doi.org/10.5194/fr-21-159-2018

\title{
Osseous anomalies of the cryptobranchid Eoscapherpeton asiaticum (Amphibia: Caudata) from the Late Cretaceous of Uzbekistan
}

\author{
Pavel Skutschas, Veniamin Kolchanov, Elizaveta Boitsova, and Ivan Kuzmin \\ Faculty of Biology, Department of Vertebrate Zoology, Saint Petersburg State University, \\ Universitetskaya nab. 7/9, 199034 Saint Petersburg, Russia \\ Correspondence: Pavel Skutschas (skutchas@mail.ru,p.skutschas@spbu.ru)
}

Received: 25 December 2017 - Revised: 20 March 2018 - Accepted: 9 April 2018 - Published: 27 April 2018

\begin{abstract}
Osseous anomalies/pathologies in the Late Cretaceous cryptobranchid salamander Eoscapherpeton asiaticum from the Turonian Bissekty Formation of Uzbekistan are analyzed using gross morphological description, microCT, and histologic analysis. These pathologies result from trauma (fractured and subsequently healed dentary and femora; hematoma on femur), possible infection due to trauma (prearticular with exostosis and necrotic cavities; anterior trunk vertebra and fused vertebra with pathological enlargements formed during reactive periosteal growth) and congenital disorders (hemivertebra and fused vertebrae with shortened asymmetrical centra and abnormal arrangements of transverse processes). The origin of the pathologies of two atlantal specimens (enlargement of transverse processes) is unclear. Our report of hemivertebra in Eoscapherpeton is the first occurrence of this congenital pathology in a fossil lissamphibian. The occurrence of several traumatic femoral pathologies in Eoscapherpeton could be a result of intraspecific aggressive behavior. Bone pathologies are described for the first time in fossil salamanders.
\end{abstract}

\section{Introduction}

The study of paleopathology offers an effective tool for understanding some aspects of vertebrate paleobiology, including lifestyle and behavior (e.g., Hanna, 2002; Martinelli et al., 2015), as well as reaction of the skeletal tissues to diseases and injuries (traumas) through the time (Rothschild and Tanke 1991; Rothschild and Martin, 2006; Waldron, 2009). Despite numerous paleopathological studies of different tetrapods (e.g., dinosaurs, crocodiles), paleopathologies among fossil members of modern groups of amphibians (i.e., lissamphibians: frogs, salamanders and caecilians) are poorly documented (Rothschild, 2012; Rothschild and Laub, 2013). Reported skeletal anomalies among fossil salamanders are limited to polydactyly, abnormal phalangeal counts and supernumerary hind limbs in the Jurassic cryptobranchid Chunerpeton tianyiensis from China (Wang et al., 2016). Other pathologies in fossil salamanders have not been described and analyzed for etiology (Rothschild et al., 2012).

Expeditions to the Kyzylkum Desert (Uzbekistan) in 1977-1994 by Lev A. Nesov and in 1997-2006 by the international Uzbek/Russian/British/American/Canadian Joint Paleontological Expeditions (URBAC) yielded several thousand bones of the fossil cryptobranchid salamander Eoscapherpeton asiaticum from the Upper Cretaceous (Turonian) Bissekty Formation (Nesov 1981, 1997; Skutschas, 2009, 2013). This abundant material contains several abnormal specimens of Eoscapherpeton asiaticum, including fused vertebrae, fractured bones and some other osseous pathologies. The main aims of this study are to describe osseous anomalies in Eoscapherpeton asiaticum (using gross morphological description, microCT, and histologic analysis) and to discuss their possible etiology.

\section{Materials and methods}

A total of 17 specimens representing isolated elements from the Dzharakuduk locality of the Turonian Bissekty Formation (Redman and Leighton, 2009) that belong to the cryptobranchid salamander Eoscapherpeton asiaticum were examined. All these specimens are stored at the Paleoherpetological collection (ZIN PH) of the Zoological Institute of the Russian Academy of Sciences, Saint Petersburg, Russia. The abnormal specimens include two bones of the lower 


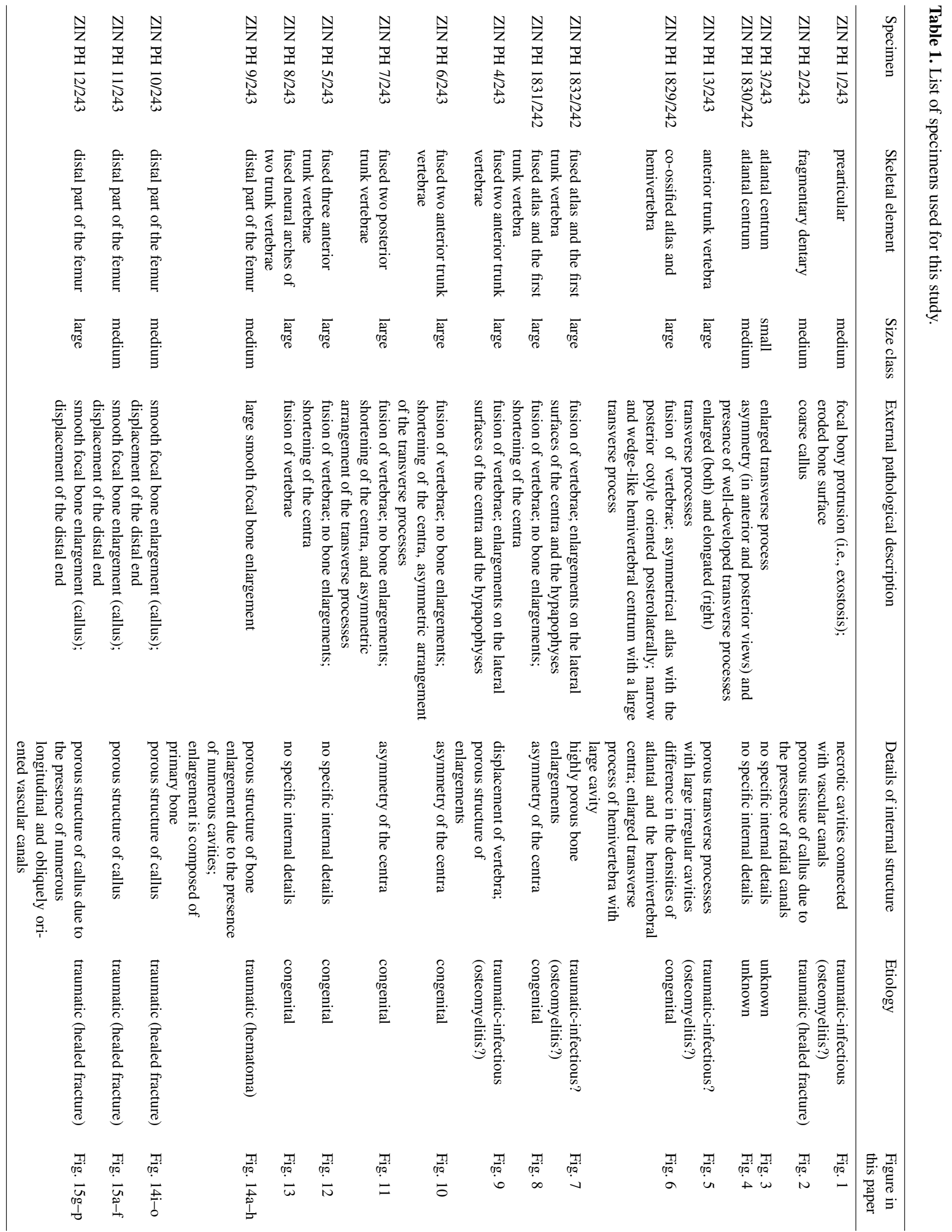




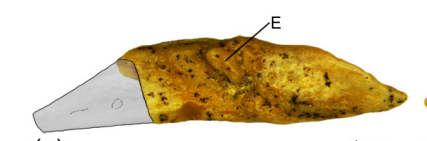

(a)

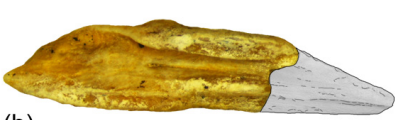

(b)

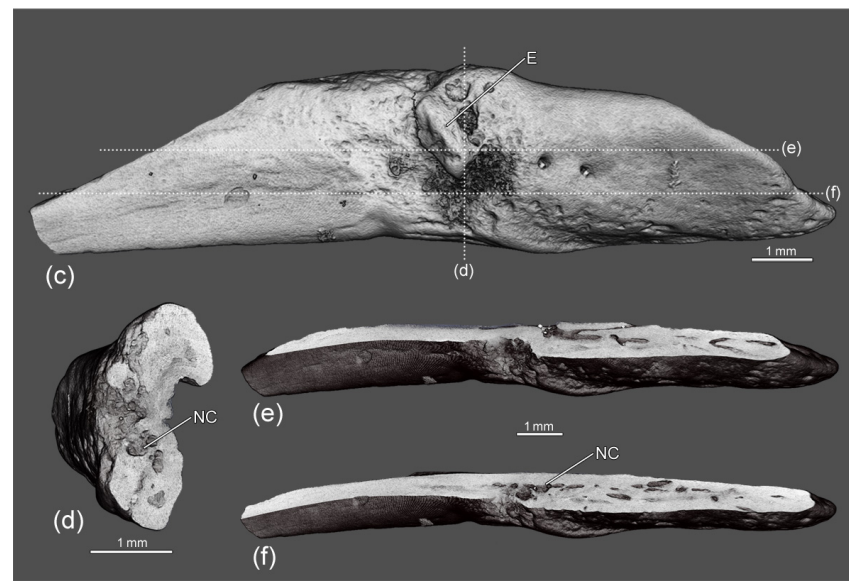

Figure 1. The prearticular ZIN PH 1/243 of Eoscapherpeton asiaticum. (a) Medial view, (b) lateral view, (c) medial view (digital restoration) with the locations of the microCT digital sections, (d) digital transverse section, and (e-f) digital longitudinal sections. Abbreviations: $\mathrm{E}$ - exostosis; $\mathrm{NC}$ - necrotic cavity.

jaw (prearticular ZIN PH 1/243, fragmentary dentary ZIN PH 2/243), two atlases (ZIN PH 3/243, ZIN PH 1830/242), trunk vertebra (ZIN PH 13/243), fused vertebrae (ZIN PH 1829/242, ZIN PH 1831/242, ZIN PH 1832/242, ZIN PH 4/243, ZIN PH 5/243, ZIN PH 6/243, ZIN PH 7/243, ZIN PH 8/243), and distal fragments of femora (ZIN PH 9/243, ZIN PH 10/243, ZIN PH 11/243, ZIN PH 12/243) (Table 1). All specimens were CT-scanned (at $100 \mathrm{kV}$ and $0.1 \mathrm{~mA}$, generating a resolution of $2.9 \mu \mathrm{m}$ of pixel size and an output of $4000 \times 4000$ pixels per slice) at the Saint Petersburg State University Research Centre for X-ray Diffraction Studies (Saint Petersburg, Russia) using a Skyscan 1172 CT scanner. CT scan data were imported to the software Amira 6.3.0 (FEI-VSG Company), where the model was reconstructed and segmented.

The CT data are deposited in the Department of Vertebrate Zoology of the Saint Petersburg State University, Saint Petersburg, Russia, and can be made available by the present authors for the purpose of scientific study.

For the histologic analysis, standard petrographic thin sections (transverse) of two abnormal femora (distal fragments ZIN PH 9/243, ZIN PH 12/243) were prepared. The sections were observed under polarized light using an optical microscope (Leica 4500, Leica Microsystems, Wetzlar, Germany) in the Saint Petersburg State University Research Centre for X-ray Diffraction Studies, Russia. Histological terminology follows Francillon-Vieillot et al. (1990). The thin sections used in study are housed in the histological collection of
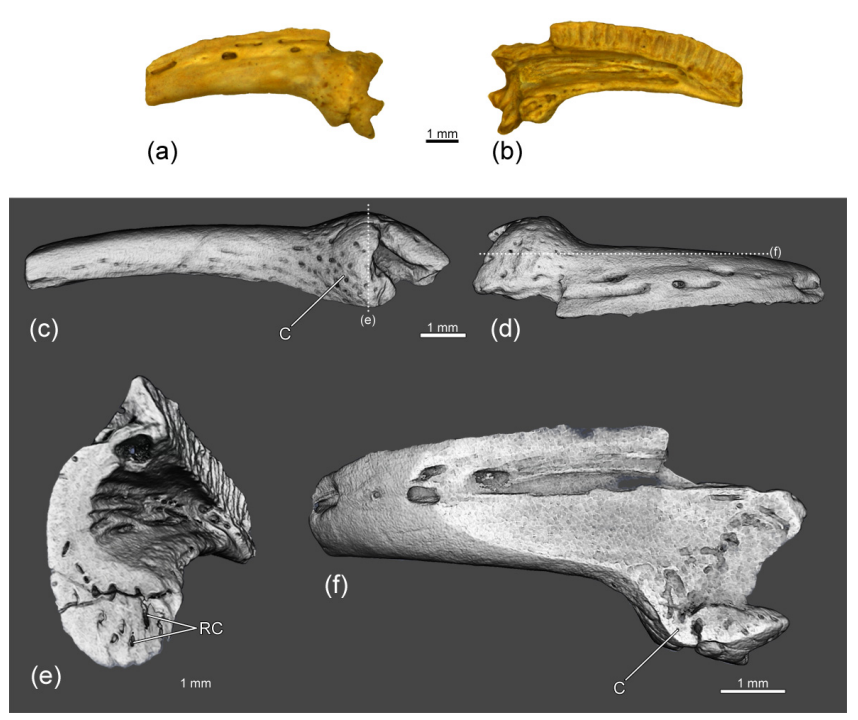

Figure 2. The dentary ZIN PH 2/243 of Eoscapherpeton asiaticum. (a) Medial view, (b) lateral view, (c) ventral view (digital restoration) with the location of the transverse microCT digital section, (d) dorsal view (digital restoration) with the location of the longitudinal microCT digital section, (e) digital transverse section, and (f) digital longitudinal section. Abbreviations: C - callus; $\mathrm{RC}$ - radial canals.

the Department of Vertebrate Zoology, Saint Petersburg State University, Saint Petersburg, Russia.

\section{Results}

\subsection{Pathologies of bones of the lower jaw}

Osseous anomalies were found in the medium-sized prearticular ZIN PH 1/243 and the fragmentary dentary ZIN PH 2/243 (Table 1). The prearticular ZIN PH 1/243 (Fig. 1) exhibits a focal bony protrusion (i.e., exostosis) on the medial surface. The bone surface around the exostosis is eroded due to the presence of relatively deep notches of irregular shape. Internal examination reveals the presence of large necrotic cavities which were connected with vascular canals (Fig. 1df) and with erosion notches on the bone surface. The possible diagnosis for this pathology is osteomyelitis - inflammation of bone and bone marrow (usually posttraumatic) caused by bacteria (more rarely by fungi), resulting in the periosteal reaction and irregular bone surface (Rothschild and Martin, 2006; Peterson and Vittore, 2012; Marais et al., 2014; García et al., 2016; Ramírez-Velasco et al., 2016 ).

The medium-sized fragmentary dentary ZIN PH 2/243 (Fig. 2) displays a focal bone enlargement (i.e., callus). Internally, the tissue of the callus is porous (in comparison to that of normal tissue of the bone) and contains several radial canals (Fig. 2e-f). The callus was formed during the healing of traumatic fracture of the dentary (traumatic nature of 
(a)

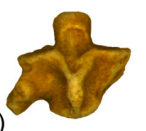

(b)

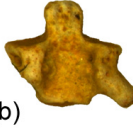

(c)
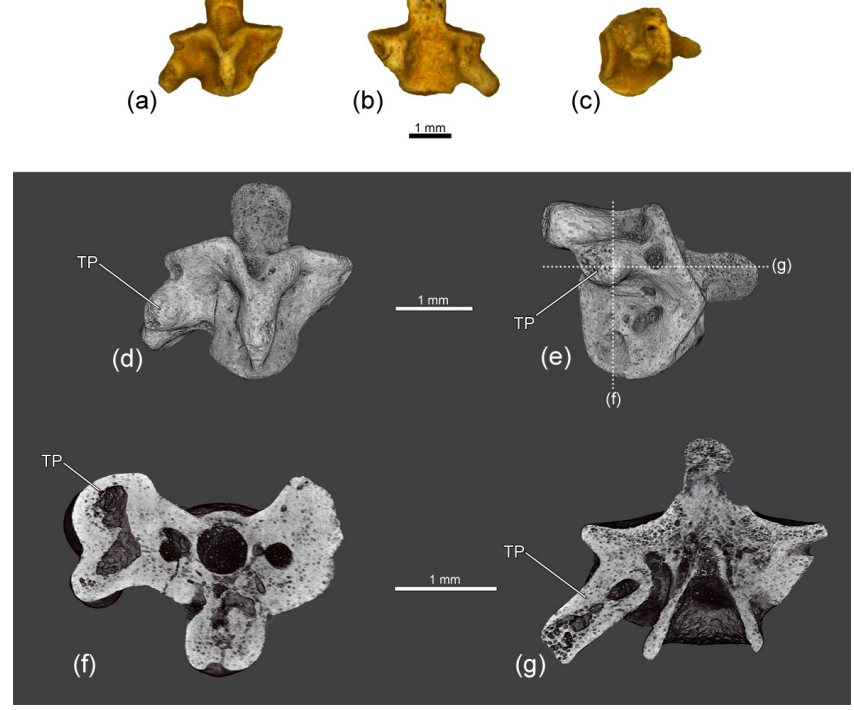

Figure 3. The atlantal centrum ZIN PH 3/243 of Eoscapherpeton asiaticum. (a) Ventral view, (b) dorsal view, (c) right lateral view, (d) ventral view (digital restoration), (e) right lateral view (digital restoration) with the locations of the microCT digital sections, (f) digital transverse section, and (g) digital longitudinal section (horizontal plane) in ventral view. Abbreviations: TP - transverse process.

pathology) (Rothschild and Martin, 2006; Pardo-Pérez et al., 2017).

\subsection{Atlantal pathologies}

Two atlantal centra in the sample show the osseous anomalies: the small centrum ZIN PH 3/243 and one medium-sized centrum ZIN PH 1830/242 (Table 1). The small atlas ZIN PH 3/243 (Fig. 3) is characterized by an enlarged transverse process (i.e., rib bearer) on the right lateral surface. The inner structure of the enlarged transverse process demonstrates the presence of a large cavity (i.e channel) that in life was likely filled by cartilage (Fig. $3 \mathrm{f}-\mathrm{g}$ ). There are no differences in porosity in the different parts of the cortical regions and no clear evidence of fractures or inflammation process. The origin of the pathology is unknown and it could be traumaticinfectious, developmental or neoplasm (Rothschild and Martin, 2006; Waldron, 2009).

The centrum of the larger atlas ZIN PH 1830/242 (Fig. 4) is asymmetric (in anterior and posterior views) and bears well-developed transverse processes on the both lateral surfaces. The inner structure of the enlarged transverse processes is similar to that in ZIN PH 3/243 (the presence of the large cavity; Fig. 4g). Like in ZIN PH 3/243, the origin of the pathology is unclear. (a)
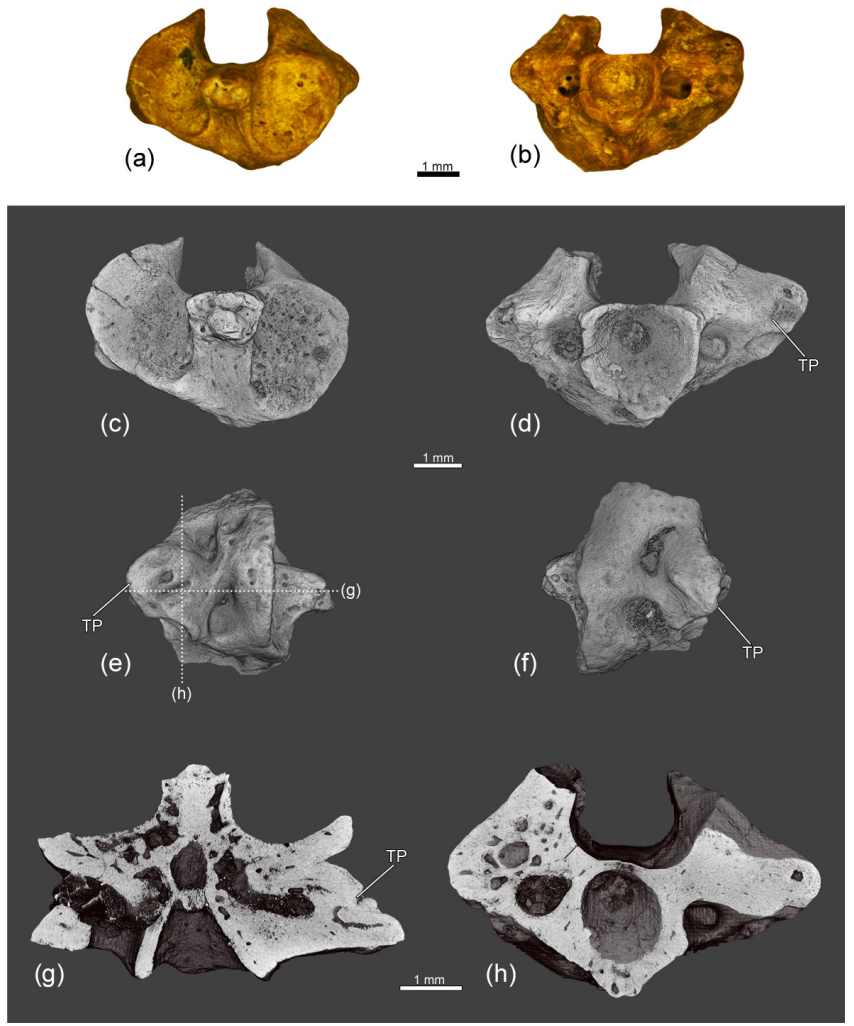

Figure 4. The atlantal centrum ZIN PH 1830/242 of Eoscapherpeton asiaticum. (a) Anterior view, (b) posterior view, (c) anterior view (digital restoration), (d) posterior view (digital restoration), (e) right lateral view (digital restoration) with the locations of the microCT digital sections, (f) left lateral view (digital restoration), (g) digital longitudinal section (horizontal plane) in dorsal view, and (h) digital transverse section in posterior view. Abbreviations: TP transverse process.

\subsection{Abnormal trunk vertebra}

The large anterior trunk vertebra ZIN PH 13/243 (Fig. 5) exhibits an abnormal structure of the transverse processes on both lateral surfaces of the centrum: right transverse processes are slightly enlarged, while left transverse processes are significantly enlarged and elongated (probably due to the fusion with a rib). The internal structure of the enlarged transverse processes is highly porous and contains large irregular cavities. Reactive bone growth is likely the result of complication of healing after trauma and the subsequent enlargement of transverse processes could be caused by osteomyelitis (traumatic-infectious origin of pathology) (Rothschild and Martin, 2006; Waldron, 2009; García et al., 2016).

\subsection{Hemivertebra}

The specimen ZIN PH 1829/242 is an asymmetrical (in dorsal and ventral view) fragmentary large atlas that is coossified with a hemivertebra (Fig. 6). The posterior cotyle 

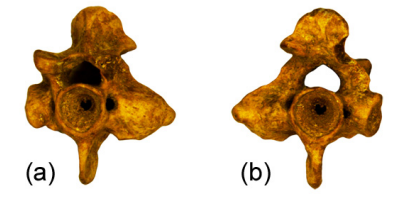

(c)

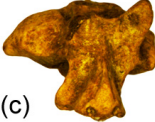

(d)
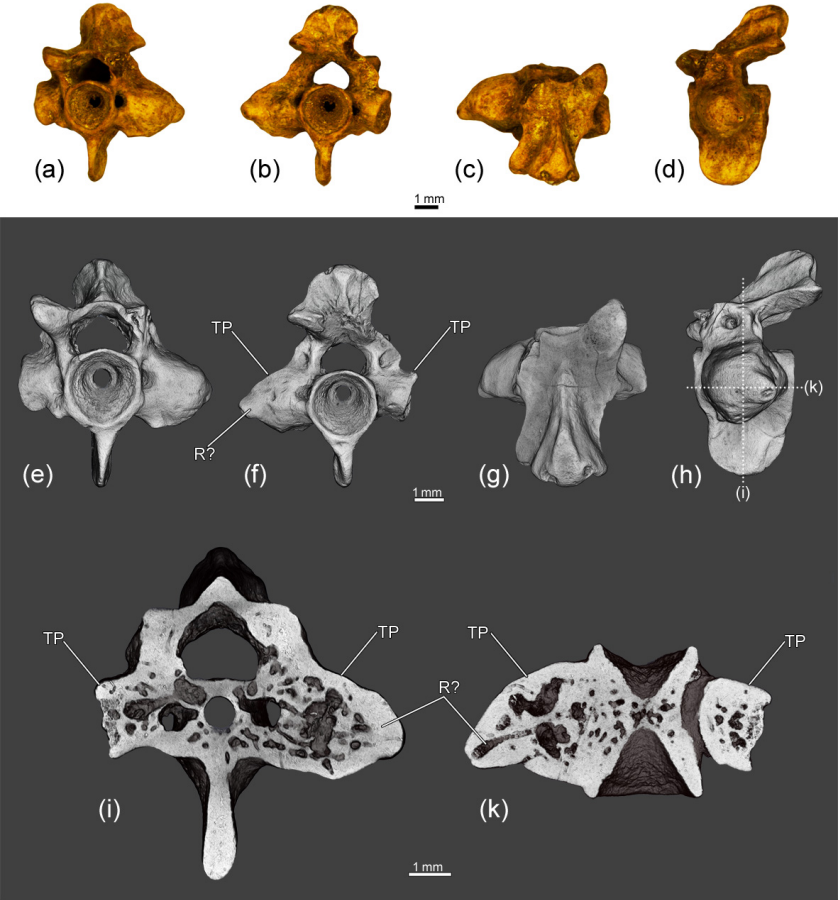

Figure 5. The anterior trunk vertebra ZIN PH 13/243 of Eoscapherpeton asiaticum. (a) Anterior view, (b) posterior view, (c) dorsal view, (d) left lateral view, (e) anterior view (digital restoration), (f) posterior view (digital restoration), (g) dorsal view (digital restoration), (h) left lateral view (digital restoration) with the locations of the microCT digital sections, (i) digital transverse section in anterior view, and (k) digital longitudinal section (horizontal plane) in dorsal view. Abbreviations: TP - transverse process; R? - rib?.

of the atlantal centrum is oriented posterolaterally. The hemivertebral centrum is narrow, wedge-like and located at the posterior left portion of the specimen. The hemivertebral centrum bears a large transverse process. The bases of neural arches of the atlas and hemivertebra are not fused. Externally, the boundary between atlantal and the hemivertebral centra is clearly traceable as a groove on the lateral surface of the hypapophysis. Internally, the boundary is not clearly evident, but some differences in the densities of atlantal and the hemivertebral centra are visible in some virtual slices (Fig. 6i). The inner structure of the enlarged transverse process demonstrates the presence of a large cavity (i.e., channel).

\subsection{Fusion of vertebrae}

The sample of fused vertebrae (all vertebrae are relatively large in size) includes two specimens of fused atlas and the first trunk vertebrae (ZIN PH 1831-1832/242), two specimens of the fused two anterior trunk vertebrae (ZIN $\mathrm{PH} 4 / 243,6 / 243$ ), one specimen of the fused three anterior trunk vertebrae (ZIN PH 5/243), one specimen of the fused two posterior trunk vertebrae (ZIN PH 7/243) and one spec-
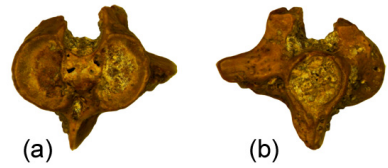

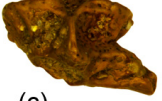

(c) $1 \mathrm{~mm}$

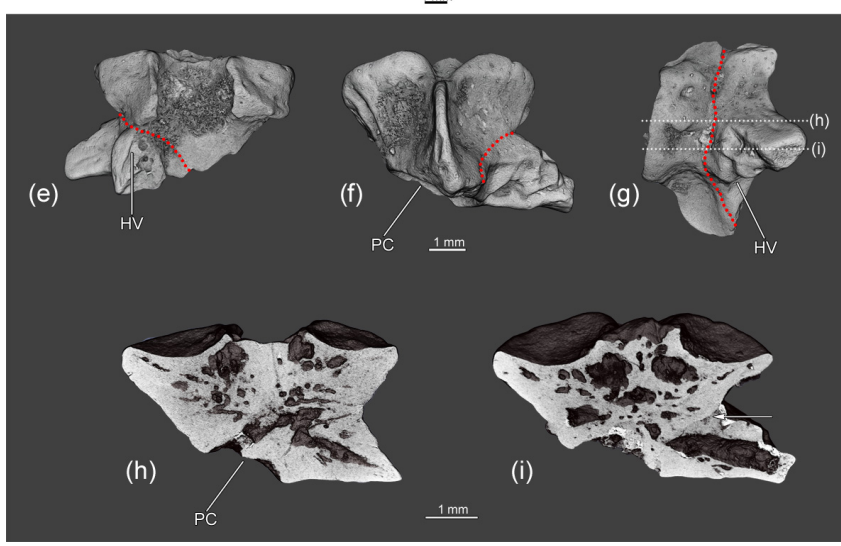

Figure 6. The co-ossified atlas and hemivertebra ZIN PH 1829/242 of Eoscapherpeton asiaticum. (a) Anterior view, (b) posterior view, (c) ventral view, (d) dorsal view, (e) dorsal view (digital restoration), (f) ventral view (digital restoration), (g) left lateral view (digital restoration) with the locations of the microCT digital sections, and (h-i) digital longitudinal sections (horizontal plane) in ventral view. Broken lines and an arrow show the border between atlas and hemivertebra. Abbreviations: HV - hemivertebra; PC - posterior atlantal cotyle.

imen of the fused neural arches of two trunk vertebrae (ZIN PH 8/243) (total number of specimens - 7; Table 1).

The specimen ZIN PH 1832/242 (Fig. 7) exhibits enlargements on the lateral surfaces of the centra and the hypapophyses along the suture between the atlas and the first trunk vertebrae that were formed during reactive bone growth. The internal structure of these enlarged areas is highly porous. The enlargements could be caused by osteomyelitis after trauma (traumatic-infectious nature of pathology).

The specimen ZIN PH 1831/242 (Fig. 8) displays the fusion of the atlas and the first trunk vertebrae without notable pathological enlargements on the lateral surfaces of the centra and the hypapophyses (only low ridge is present along the suture between atlas and the first trunk vertebrae). This suggests the absence of the periosteal reactive bone growth. Both vertebrae are shortened in comparison with normal vertebrae or that in the specimen (ZIN PH 1832/242). The absence of any traces of trauma (e.g., fracture, displacement, callus) and inflammation of bone (e.g., enlargements formed during reactive bone growth, necrotic cavities) suggests the congenital origin of the fusion.

The fused two anterior trunk vertebrae ZIN PH 4/243 (Fig. 9) are characterized by the enlargements on the lateral surfaces of the centra and the hypapophyses along the suture between vertebrae and by deformation of the hypapophyses. 
(a)

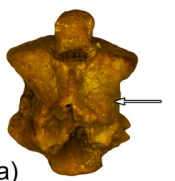

(b)

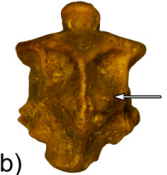

$1 \mathrm{~mm}$ (c)

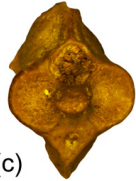

(a)

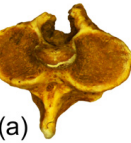

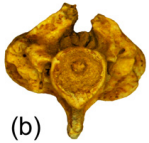

(c)

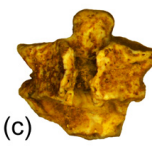

$1 \mathrm{~mm}$

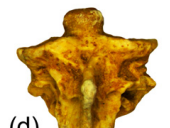

(d)

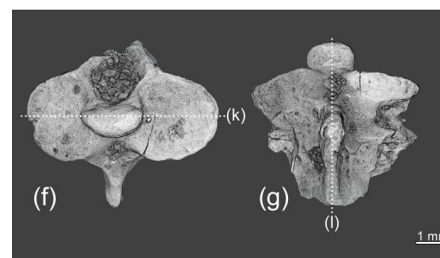

(h)

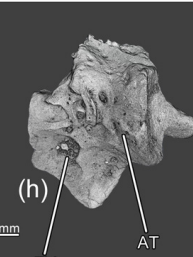

(e)

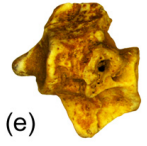

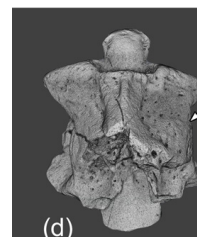

(e)

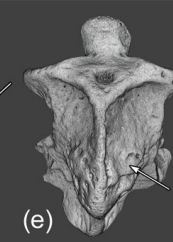

(f)

$1 \mathrm{~mm}$
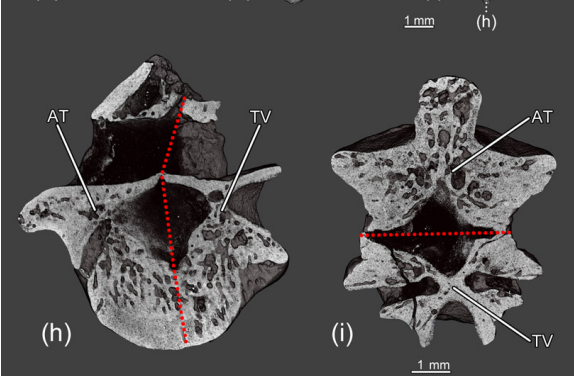

(i)

(g)
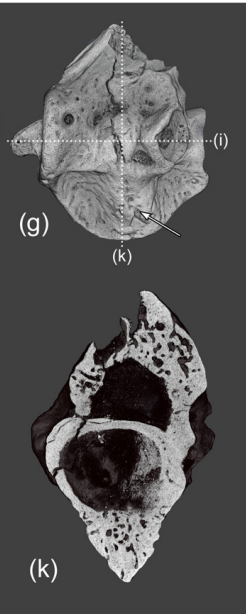

Figure 7. The fused atlas and the first trunk vertebra ZIN PH 1832/242 of Eoscapherpeton asiaticum. (a) Dorsal view, (b) ventral view, (c) anterior view, (d) dorsal view (digital restoration), (e) ventral view (digital restoration), (f) anterior view (digital restoration) with the location of longitudinal the microCT digital section, (g) left lateral view (digital restoration) with the locations of the microCT digital sections, (h) digital longitudinal section (vertical plane), (i) digital longitudinal section (horizontal plane) in dorsal view, and (k) digital transverse section in posterior view. Broken lines show the border between atlas and trunk vertebra. Arrows show bone enlargements. Abbreviations: AT - atlas; TV - trunk vertebra.

CT data show that the more posterior vertebra is slightly displaced in relation to the more anterior vertebrae (Fig. 91), so the trauma took place before the forming of pathological enlargements. The internal structure of enlarged areas is highly porous and contains large necrotic cavities.

The fused two anterior trunk vertebrae ZIN PH 6/243 (Fig. 10) are characterized by shortening of the centra, asymmetry of the centra (due to the unequal development of the anterior and posterior portions) and asymmetric arrangement of the transverse processes (widely separated on the left side and closely spaced on the right side of the fused centra). The bases of neural arches are fused and a large spinal nerve foramen is present.

Similarly to the specimen ZIN PH 6/243, the fused two posterior trunk vertebrae ZIN PH 7/243 (Fig. 11) exhibit the vertebral shortening with asymmetry of the centra and asymmetric arrangement of the transverse processes. The absence of any traces of trauma and inflammation of bone suggests the congenital origin of the fusion. The asymmetric arrangement of the transverse processes and vertebral shortening

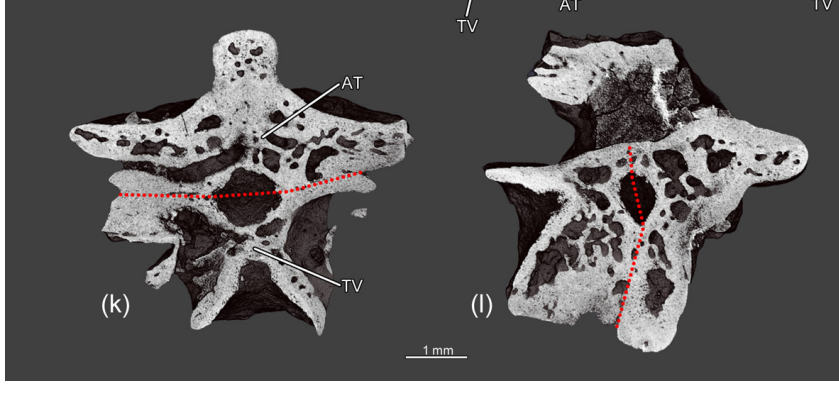

Figure 8. The fused atlas and the first trunk vertebra ZIN PH 1831/242 of Eoscapherpeton asiaticum. (a) anterior view, (b) posterior view, (c) dorsal view, (d) ventral view, (e) left lateral view, (f) anterior view (digital restoration) with the location of longitudinal the microCT digital section, (g) ventral view (digital restoration) with the location of longitudinal the microCT digital section, (h) right lateral view (digital restoration), (i) left lateral view (digital restoration), (k) digital longitudinal section (horizontal plane) in dorsal view, and (l) digital longitudinal section (vertical plane). Broken lines show the border between atlas and trunk vertebra. Abbreviations: AT - atlas; TV - trunk vertebra.

could be the result of abnormal segmentation during embryogenesis.

The specimen ZIN PH 5/243 consists of three fused anterior trunk vertebrae (Fig. 12). The vertebrae exhibit the vertebral shortening and do not display any signs of trauma and/or inflammation of bone. We suggest the congenital origin of the fusion in ZIN PH 5/243.

The neural arches of two trunk vertebrae ZIN PH 8/243 (Fig. 13) are fused without any trace of suture. The more anterior neural arch is lower than the more posterior one. There are no pathological enlargements (no signs of periosteal reactive growth), and the fusion of these neural arches is most likely of congenital origin.

\subsection{Femoral pathologies}

The distal fragment of the medium-sized femur ZIN PH 9/243 (Fig. 14a-h) displays the presence of large smooth focal bone enlargement (i.e., periosteal elevation) in the metaphyseal region. The internal structure of this bone enlargement is porous due to the presence of large cavities of irregular shape (i.e., erosion bays) (Fig. 14f-h). The tissue 

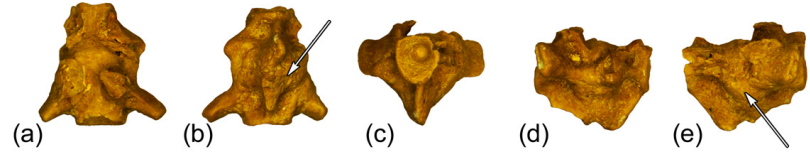

(a)

(b)

$1 \underline{\mathrm{mm}}$

(d)

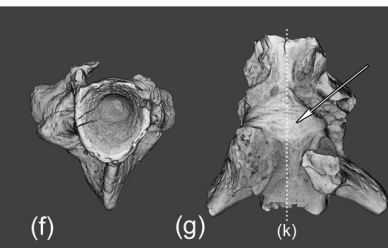

(f)

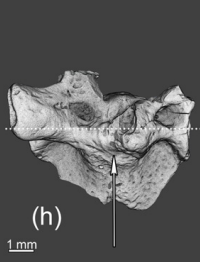

(h)

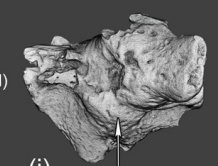

(i)

(k)

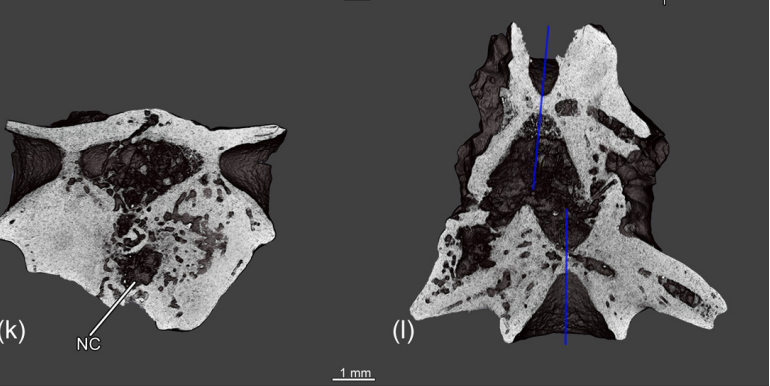

Figure 9. The fused two anterior trunk vertebrae ZIN PH 4/243 of Eoscapherpeton asiaticum. (a) Dorsal view, (b) ventral view, (c) anterior view, (d) right lateral view, (e) left lateral view, (f) anterior view (digital restoration), (g) dorsal view (digital restoration) with the location of longitudinal the microCT digital section, (h) right lateral view (digital restoration) with the location of longitudinal the microCT digital section, (i) left lateral view (digital restoration), (k) digital longitudinal section (vertical plane), and (l) digital longitudinal section (horizontal plane) in dorsal view. Blue lines show the original median line of the centra prior to the trauma. Arrows show bone enlargements. Abbreviations: $\mathrm{NC}$ - necrotic cavity.

of the enlargement is entirely composed of a parallel-fibered primary bone of periosteal origin. The parallel-fibered primary bone in the outer part of the enlargement is more organized than in the inner porous part and is similar to that of the normal cortical bone of this femur (Fig. 14h). No evidence of fracture was revealed by CT scanning and histological analysis. Similar bone enlargement in modern reptiles was identified as hematoma (Rothschild et al., 2010, 2012). We also interpret the femoral pathology on ZIN PH 9/243 as hematoma that could have been caused by trauma (traumatic origin).

All other femoral specimens in the sample (two distal fragments ZIN PH 10-11/243 of medium-sized femora and one distal fragment ZIN PH 12/243 of large femora; Table 1, Figs. 14i-o, 15) display the signs of healed fractures that are traceable in CT images. Externally, all the specimens demonstrate displacements of the distal end of the bone and have large smooth focal bone enlargements (i.e., calluses) in the metaphyseal region that were formed during the healing of traumatic fractures. The internal structure of the calluses is porous and contains numerous longitudinal and obliquely

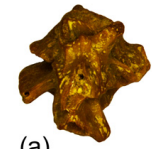

(a)
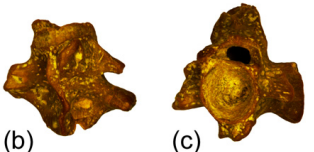

(c)

(d)

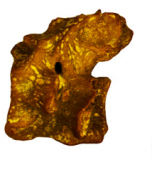

(e)
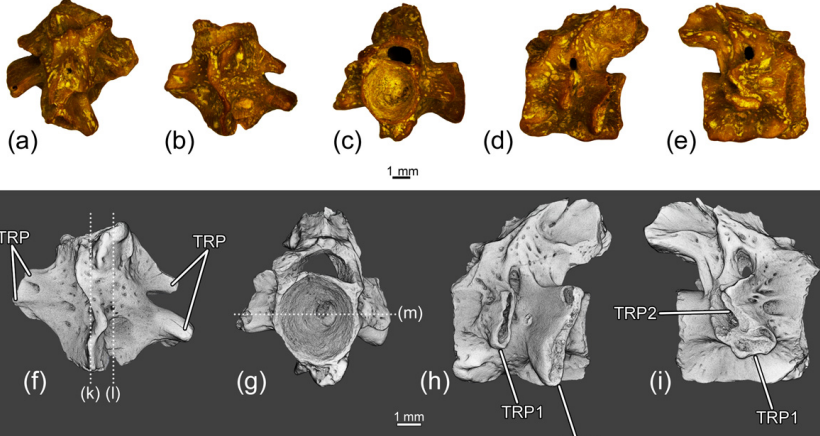

m

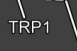

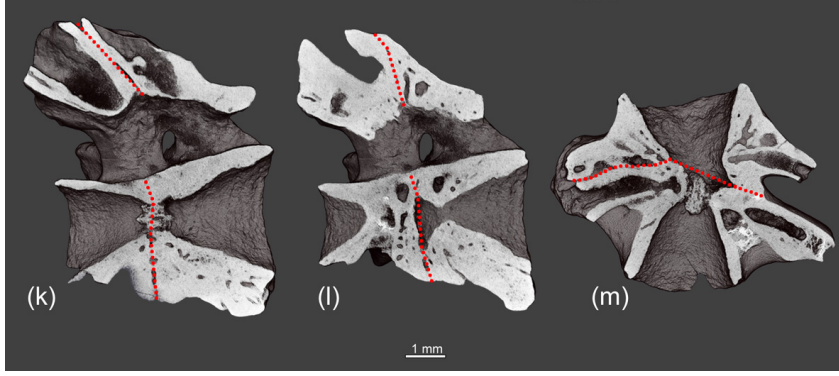

Figure 10. The fused two anterior trunk vertebrae ZIN PH 6/243 of Eoscapherpeton asiaticum. (a) Dorsal view, (b) ventral view, (c) anterior view, (d) left lateral view, (e) right lateral view, (f) ventral view (digital restoration) with the location of longitudinal the microCT digital sections, (g) anterior view (digital restoration) with the location of longitudinal the microCT digital section, (h) left lateral view (digital restoration), (i) right lateral view (digital restoration), (k-l) digital longitudinal sections (vertical plane), and (m) digital longitudinal section (horizontal plane) in ventral view. Broken lines show the border between trunk vertebrae. Abbreviations: NA - neural arch; TP1 - transverse process of more anterior vertebra; TP2 - transverse process of more posterior vertebra.

oriented vascular canals that are typical for these structures (Fig. 15n-p).

\section{Discussion}

\subsection{General discussion}

Eoscapherpeton asiaticum demonstrates a wide spectrum of osseous anomalies/pathologies, resulting from trauma, possible infection due to trauma, and congenital disorders. Traumatic origins of pathologies are suggested for the dentary ZIN PH 2/243 (the presence of the fracture and the callus) and for femoral fragments ZIN PH 9-12/243 (hematoma and healed fractures with the calluses on the distal portions of the bones) (Table 1). The possible traumatic-infectious pathologies are associated with bone enlargements formed during reactive bone growth and/or the presence of necrotic cavities are suggested for the prearticular ZIN PH 1/243, the anterior trunk vertebra ZIN PH 13/243, the fused atlas and the first trunk vertebrae ZIN PH 1832/242, and the fused two ante- 
(a)

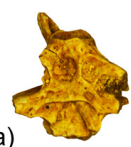

(b)

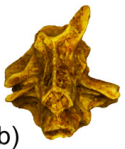

(c)

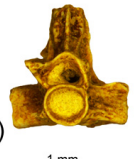

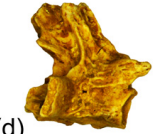

(d)
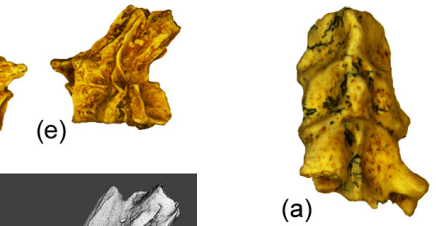

(a)

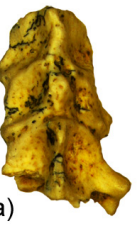

(b)

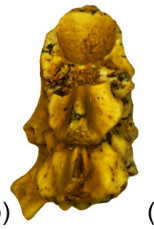

(c)

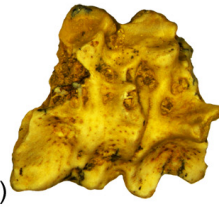

$1 \mathrm{~mm}$
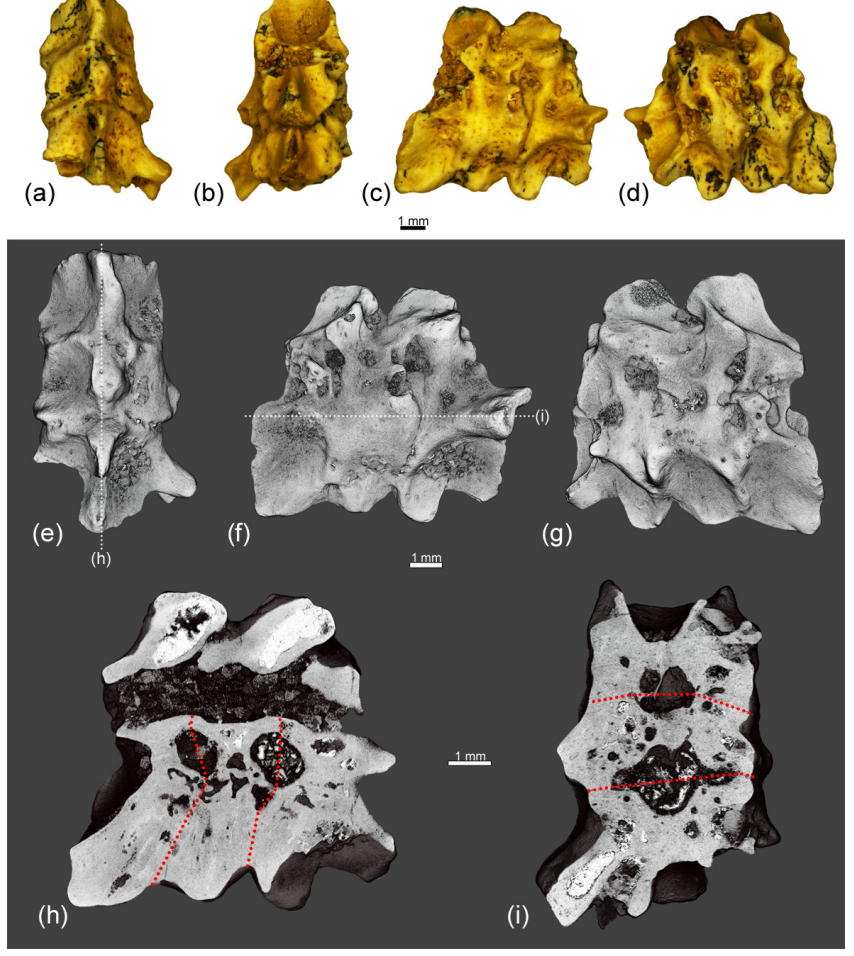

Figure 12. The fused three anterior trunk vertebrae ZIN PH 5/243 of Eoscapherpeton asiaticum. (a) Ventral view, (b) dorsal view, (c) left lateral view, (d) right lateral view, (e) ventral view (digital restoration) with the location of longitudinal the microCT digital section, (f) left lateral view (digital restoration) with the location of longitudinal the microCT digital section, (g) right lateral view (digital restoration), (h) digital longitudinal section (vertical plane), and (i) digital longitudinal section (horizontal plane) in dorsal view. Broken lines show the border between trunk vertebrae.

rior trunk vertebrae ZIN PH 4/243 (Table 1). The congenital pathologies of E. asiaticum are represented by the hemivertebra (specimen ZIN PH 1829/24; see discussion below) and by fusion of two (fused atlas and anterior trunk vertebra ZIN PH 1831/242, fused anterior trunk vertebrae ZIN PH 6/243, fused posterior trunk vertebrae ZIN PH 7/243 and for fused neural arches 8/243) or three (fused anterior trunk vertebrae ZIN PH 5/243) vertebrae (Table 1). The total number of these vertebral congenital pathologies in E. asiaticum is very low $(n=5)$ in comparison with thousands of collected vertebrae of this salamander (only collection of atlases ZIN PH 242 contains 1909 specimens, while there are only two specimens of congenital pathologies with atlas - ZIN PH 1829/24 and ZIN PH 1831/242. This frequency of vertebral congenital pathologies in E. asiaticum (for congenital pathologies with atlas $<0.1 \%$ ) is in accordance with the previous estimations for Paleozoic-Mesozoic temnospondyls and Holocene frogs (Rothschild and Laub, 2013; Witzmann et al., 2013).

\subsection{Hemivertebra in Eoscapherpeton asiaticum}

A hemivertebra is a congenital malformation in which one or more lateral halves of vertebrae are intercalated between neighboring vertebrae. Formation of hemivertebrae is a result of abnormal segmentation during embryogenesis (i.e., failure of somitogenesis before chondrification and ossification of the vertebral anlagen; e.g., Witzmann et al., 2008, 2013; Burnham et al., 2013). In amniots, single vertebrae are derived from the sclerotome of two adjacent somites and this process is widely known as resegmentation (Piekarski and Olsson, 2014, and references therein). Resegmentation have been proposed to be present in all modern lissamphibians, but embryological and molecular evidence for this process was provided only for one salamander species - ambystomatid Ambystoma mexicanum (Mexican axolotl) (Piekarski and Olsson, 2014). The development of the described hemivertebra in more basal cryptobranchid salamander Eoscapherpeton asiaticum can also be explained by a failure of resegmentation. If this is correct, then it is significant for the fol- 
(a)
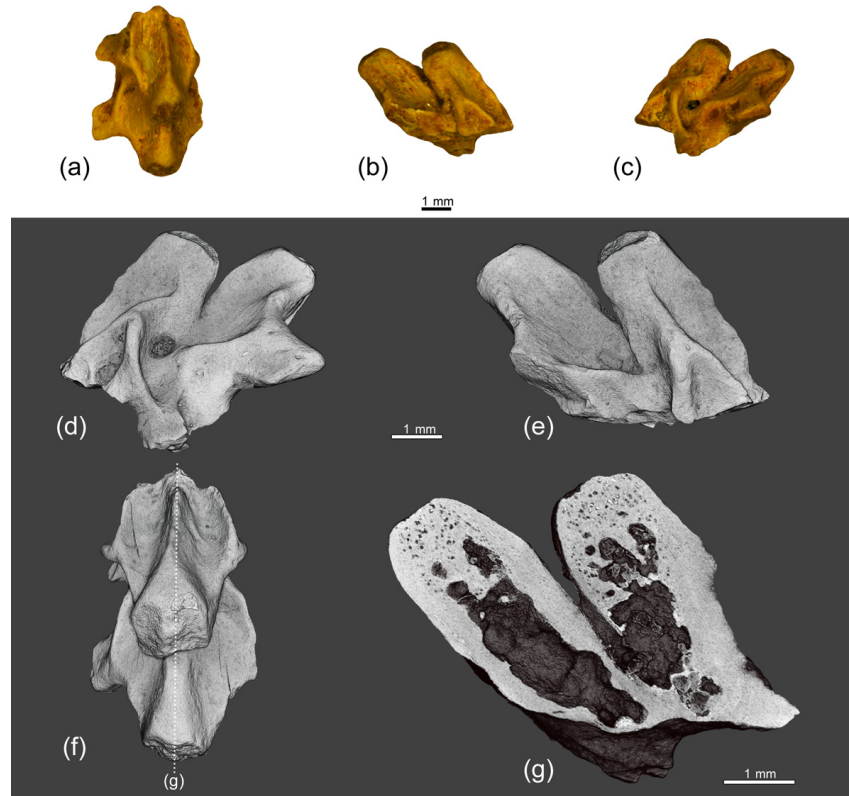

Figure 13. The fused neural arches of two trunk vertebrae ZIN PH 8/243 of Eoscapherpeton asiaticum. (a) Dorsal view, (b) right lateral view, (c) left lateral view, (d) left lateral view (digital restoration), (e) right lateral view (digital restoration), (f) dorsal view (digital restoration) with the location of longitudinal the microCT digital section, and (g) digital longitudinal section (vertical plane).

lowing reasons: (1) it is the oldest occurrence of resegmentation in salamanders, and (2) it is evidence that resegmentation is a common process for salamanders (including relatively basal cryptobranchids and advanced ambystomatids).

A hemivertebra in E. asiaticum was found in a relatively large (according to the large size of the atlas that was coossified with hemivertebra) and, presumably, adult individual (Fig. 6). The presence of a hemivertebra in a large individual suggests that this pathology did not cause its death.

The hemivertebrae have been reported earlier for some Paleozoic and Mesozoic temnospondyls (Witzmann et al., 2013). The finding of hemivertebra in the Late Cretaceous cryptobranchid E. asiaticum is the oldest (and the first described) occurrence of this pathology among modern groups of amphibians (i.e., lissamphibians: salamanders, frogs and caecilians).

\subsection{Femoral pathologies in Eoscapherpeton: possible insight into behavior}

The traumatic origin of pathologies on the distal parts of the femora (Figs. 14-15) could be evidence of frequent occurrence of hind limb injuries in Eoscapherpeton asiaticum. In modern cryptobranchids such as Cryptobranchus, limb injuries are the most common physical abnormalities in wild populations (Miller and Miller, 2005). The proposed main cause of limb injuries in Cryptobranchus is intraspecific ag-

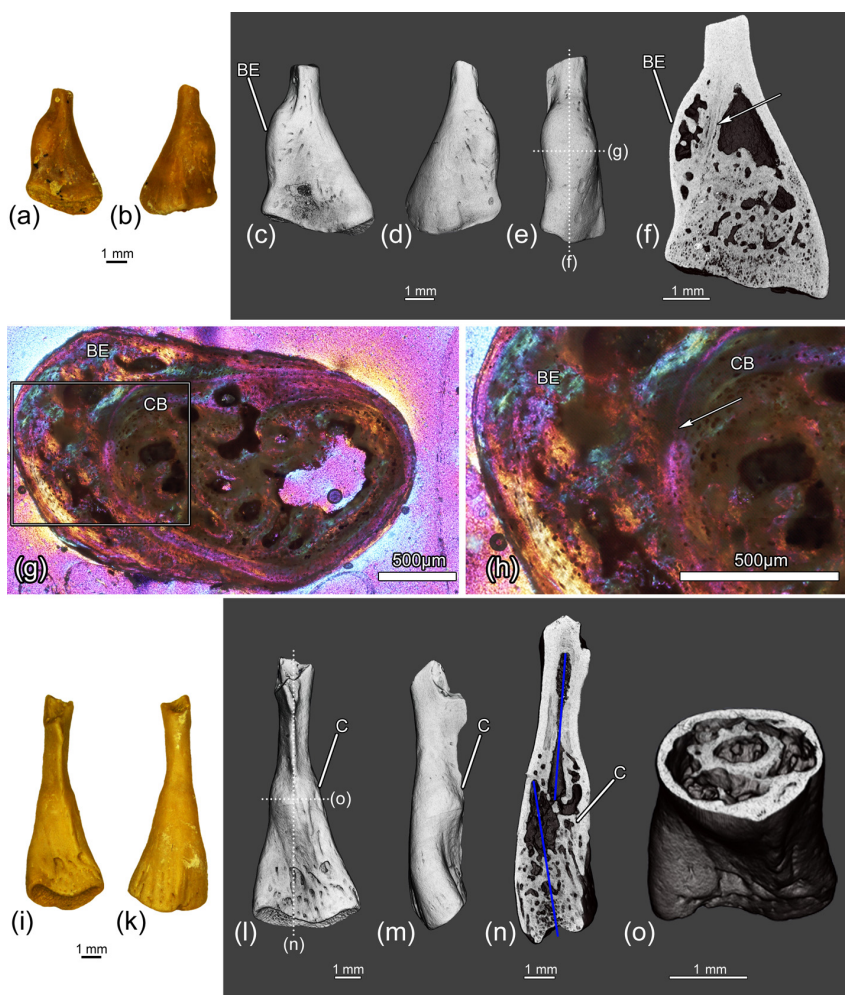

Figure 14. The distal parts of the femora ZIN PH 9/243 (a-h) and ZIN PH 10/243 (i-o) of Eoscapherpeton asiaticum. Specimen ZIN PH 9/243: (a) ventral view, (b) dorsal view, (c) ventral view (digital restoration), (d) dorsal view (digital restoration), (e) anterior or posterior view (digital restoration) with the locations of transverse histological sections and longitudinal the microCT digital section, (f) digital longitudinal section, and ( $\mathbf{g}-\mathbf{h})$ histological transverse section. The arrow shows the border between cortical bone and pathological tissue. Specimen ZIN PH 10/243: (i) ventral view, (k) dorsal view, (l) ventral view (digital restoration) with the locations of the microCT digital sections, $(\mathbf{m})$ anterior or posterior view (digital restoration), (n) digital longitudinal section, and (o) digital transverse section. Blue lines show the original bone position prior to the break. Abbreviations: $\mathrm{BE}$ - bone enlargement; $\mathrm{C}$ - callus; $\mathrm{CB}$ - cortical bone.

gression during the reproductive season (e.g., defending of nesting sites), because fresh injuries were found only during the reproductive season (namely in August and September) (Hiler et al., 2005; Miller and Miller, 2005). Taking into account the phylogenetic position of Eoscapherpeton as a member of the cryptobranchid clade and their aquatic lifestyle (see Skutschas, 2009, 2013), the reason for the frequent occurrence of hind limb injuries in E. asiaticum could be the same (intraspecific aggression during reproductive season), and this behavioral element could have appeared early in the evolution of cryptobranchids, i.e., at least in the Late Cretaceous. 


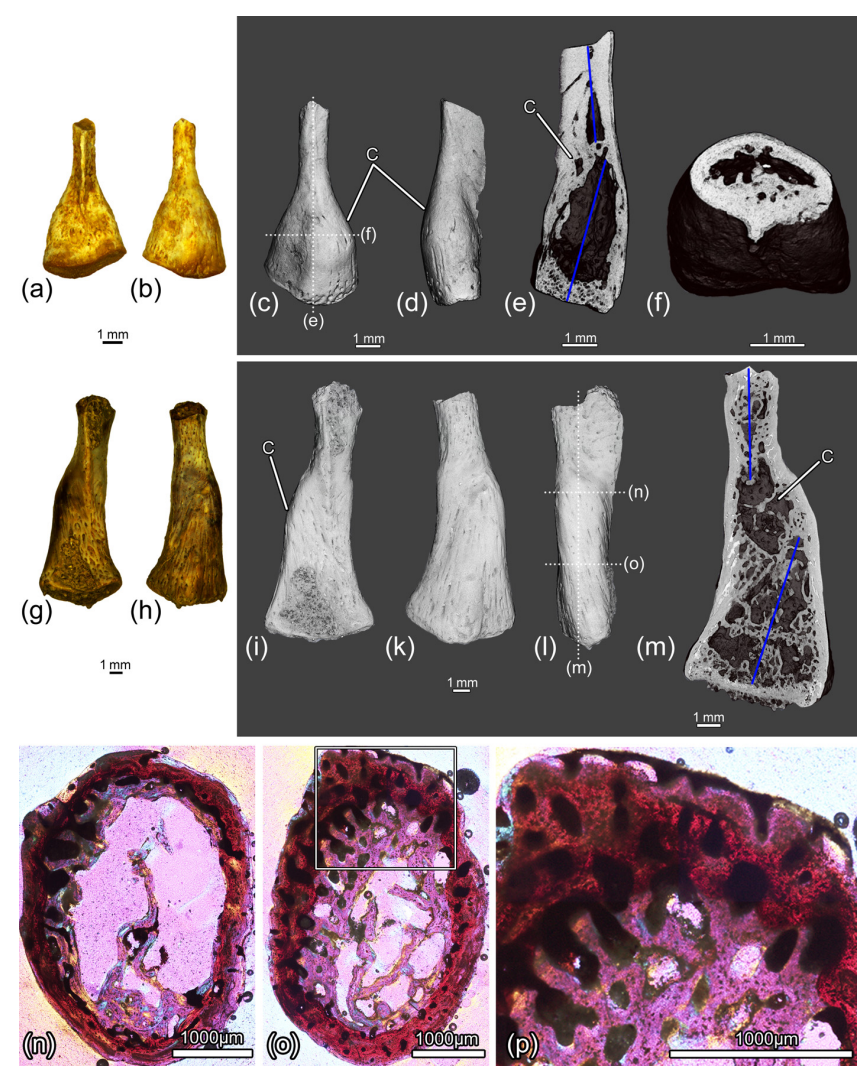

Figure 15. The distal parts of the femora ZIN PH 11/243 (a-f) and ZIN PH 12/243 (g-p) of Eoscapherpeton asiaticum. Specimen ZIN PH 11/243: (a) ventral view, (b) dorsal view, (c) dorsal view (digital restoration) with the locations of the microCT digital sections, (d) anterior or posterior view (digital restoration), (e) digital longitudinal section, and (f) digital transverse section. Specimen ZIN PH 12/243: (g) ventral view, (h) dorsal view, (i) ventral view (digital restoration), (k) dorsal view (digital restoration), (l) anterior or posterior view (digital restoration) with the locations of transverse histological sections and longitudinal the microCT digital section, (m) digital longitudinal section, and (n-p) transverse histological sections. Blue lines show the original bone position prior to the break. Abbreviations: $\mathrm{C}$ - callus.

\section{Conclusions}

The study revealed diverse osseous anomalies/pathologies in Eoscapherpeton asiaticum, which were interpreted as traumatic, possible traumatic-infectious and congenital in origin, pertaining to bones of the lower jaw, vertebrae and femora. The hemivertebra and fused vertebrae (without any traces of trauma and inflammation) document the earliest occurrence of vertebral congenital pathologies in crown-group salamanders. The frequency of vertebral congenital pathologies in $E$. asiaticum is low and in accordance with the previous estimations for temnospondyls and frogs. The numerous traumatic pathologies of femora (hematoma and healed fractures) suggest the survival of accidents and possibly the presence of in- traspecific aggression during the reproductive season in this salamander (like in modern cryptobranchids).

Data availability. Micro-CT data are archived at the Department of Vertebrate Zoology of the Saint Petersburg State University (Saint Petersburg, Russia) and can be accessed through the present authors for the purpose of scientific study.

Author contributions. PS designed the project, PS and VK performed the research, VK and IK prepared 3-D reconstructions, VK and EB prepared figures, and PS wrote the manuscript. All authors commented on the manuscript.

Competing interests. The authors declare that they have no conflict of interest.

Acknowledgements. We are grateful to the staff of the Saint Petersburg State University Research Centre for X-ray Diffraction Studies (Saint Petersburg, Russia) for their help with using the Leica 2500P microscope and for CT scanning of specimens. The authors thank Judith Pardo-Pérez and Nadia Fröbisch for providing helpful comments that improved the quality of the manuscript. This study was fulfilled under support of the Russian Scientific Fund project 14-14-00015.

Edited by: Constanze Bickelmann

Reviewed by: Nadia Fröbisch and Judith Pardo Perez

\section{References}

Burnham, D. A., Rothschild, B. M., Babiarz, J. P., and Martin, L. D.: Hemivertebrae as pathology and as a window to behavior in the fossil record, Palarch's Journal of Vertebrate Palaeontology, 10, 1-6, 2013.

Francillon-Vieillot, H., de Buffrénil, V., Castanet, J., Geraudie, J., Meunier, F., Sire, J. Y., Zylenberberg, L., and de Ricqles, A. D.: Microstructure and mineralization of vertebrate skeletal tissues, in: Skeletal biomineralization: Patterns, Processes, and Evolutionary Trends, Vol. 1, edited by: Carter, J. G., Van Nostrand Reinhold, New York, 471-530, 1990.

García, R. A., Cerda, I. A., Heller, M., Rothschild, B. M., and Zurriaguz, V.: The first evidence of osteomyelitis in a sauropod dinosaur, Lethaia, 50, 227-236, https://doi.org/10.1111/let.12189, 2016

Hanna, R. R.: Multiple injury and infection in a sub adult theropod dinosaur Allosaurus fragilis with comparisons to allosaur pathology in Cleveland-Lloyd Dinosaur Quarry Collection, J. Vertebr. Paleontol., 22, 76-90, https://doi.org/10.1671/02724634(2002)022[0076:MIAIIA]2.0.CO;2, 2002.

Hiler, W. R., Wheeler B. A., and Trauth, S. E.: Abnormalities in the Ozark Hellbender (Cryptobranchus alleganiensis bishopi) in Arkansas: A Comparison between Two Rivers with a Historical 
Perspective, Journal of the Arkansas Academy of Science, 59, 88-94, 2005.

Marais, L. C., Ferreira, N., Aldous, C., and Le Roux, T. L. B.: The classification of chronic osteomyelitis, South African Orthopoedic Journal, 13, 22-28, 2014.

Martinelli, A. G., Teixeira, V. P. A., Marinho, T. S., Fonseca, P. H. M., Cavellani, C. L., Araujo, A. J. G., Ribeiro, L. C. B., and Ferraz, M. L. F.: Fused mid-caudal vertebrae in the titanosaur Uberabatitan ribeiroi from the Late Cretaceous of Brazil and other bone lesions, Lethaia, 48, 456-462, https://doi.org/10.1111/let.12117, 2015.

Miller, B. T. and Miller J. R.: Prevalence of physical abnormalities in Eastern Hellbender (Cryptobranchus alleganiensis alleganiensis) populations of Middle Tennessee, Southeast. Nat., 4, 513-520, 2005

Nesov, L. A.: Cretaceous salamanders and frogs of Kizylkum Desert (in Russian), Trudy Zoologicheskogo Instituta AN SSSR, 101, 57-88, 1981.

Nesov, L. A.: Cretaceous nonmarine vertebrates of Northern Eurasia, Posthumous, edited by: Golovneva, L. B. and Averianov, A. O., Izdatel'stvo Sankt-Peterburgskogo Universiteta, Saint Petersburg, 218 pp., 1997 (in Russian).

Pardo-Pérez, J. M., Kear, B. P., Gómez, M., Moroni, M., and Maxwell, E. E.: Ichthyosaurian palaeopathology: evidence of injury and disease in fossil "fish lizards", J. Zool., 304, 21-33, 2017.

Peterson, J. E. and Vittore, C. P.: Cranial pathologies in a specimen of Pachycephalosaurus, PLoS One, 7, e36227, https://doi.org/10.1371/journal.pone.0036227, 2012.

Piekarski, N. and Olsson, L.: Resegmentation in the Mexican Axolotl, Ambystoma Mexicanum, J. Morphol., 275, 141-152, 2014.

Ramírez-Velasco, A. A., Morales, E., Rivera, R. H., and Tanke, D.: Spinal and rib osteopathy in Huehuecanauhtlus tiquichensis (Ornithopoda: Hadrosauroidea) from the Late Cretaceous in Mexico, Hist. Biol., 29, 208-222, https://doi.org/10.1080/08912963.2016.1147033, 2016.

Redman, C. M. and Leighton, L. R.: Multivariate faunal analysis of the Turonian Bissekty Formation: variation in the degree of marine influence in temporally and spatially averaged fossil assemblages, Palaios, 24, 18-26, 2009.
Rothschild, B. M.: Macroscopic recognition of non-traumatic osseous pathology in the post-cranial skeletons of crocodilians and lizards, J. Herpetol., 44, 13-20, https://doi.org/10.1670/08243.1, 2010.

Rothschild, B. M. and Laub, R. S.: Epidemiology of anuran pathology in the holocene component of the Hiscock site: rare or not survived, J. Herpetol., 47, 169-173, 2013.

Rothschild, B. M. and Martin, L. D.: Skeletal impact of disease, Bull. New Mexico Mus. Natl. Hist., 33, 1-230, 2006.

Rothschild, B. M. and Tanke, D.: Paleopathology of vertebrates: insights to lifestyle and health in the geological record, Geosci. Can., 19, 72-82, 1991.

Rothschild, B. M., Schultze, H.-P., and Pellegrini, R.: Herpetological Osteopathology. Annotated Bibliography of Amphibians and Reptiles, Heidelberg, Springer, 450 pp., 2012.

Skutschas, P. P.: Re-evaluation of Mynbulakia Nesov, 1981 (Lissamphibia: Caudata) and description of a new salamander genus from the Late Cretaceous of Uzbekistan, J. Vertebr. Paleontol., 29, 1-6, 2009.

Skutschas, P. P.: Mesozoic salamanders and albanerpetontids of Middle Asia, Kazakhstan, and Siberia, in: Mesozoic and Cenozoic lissamphibian and squamate assemblages of Laurasia, edited by: Gardner, J. D. and Nydam, R. L., Palaeobiodiversity and Palaeoenvironments, 93, 444-457, 2013.

Waldron, T.: Palaeopathology, Cambridge Manuals in Archaeology, Cambridge University Press, Cambridge, 279 pp., 2009.

Wang, Y., Dong, L., and Evans, S. E.: Polydactyly and other limb abnormalities in the Jurassic salamander Chunerpeton from China, Palaeobio. Palaeoenv., 96, 49-59, 2016.

Witzmann, F., Asbach, P., Remes, K., Hampe, O., Hilger, A. and Paulke, A.: Vertebral pathology in an ornithopod dinosaur: a hemivertebra in Dysalotosaurus lettowvorbecki from the Jurassic of Tanzania, Anat. Rec., 291, 1149-1155, 2008.

Witzmann, F., Rothschild, B. M., Hampe, O., Sobral, G., Gubin, Y. M., and Asbach, P.: Congenital malformations of the vertebral column in ancient amphibians, Anat. Histol. Embryol., 43, 90102, 2013. 